\title{
PENSAR LA ACCIÓN COLECTIVA EN EL CONTEXTO DE LA LOGÍSTICA HUMANITARIA: LAS LECCIONES DEL SISMO DE PISCO
}

\author{
TO PONDER ON THE COLLECTIVE ACTIONS IN THE \\ CONTEXT OF HUMANITARIAN LOGISTICS: \\ LESSONS FROM THE EARTHQUAKE IN PISCO
}

\author{
Jérôme Chandes ${ }^{1}$ y Gilles Paché ${ }^{2}$
}

\section{RESUMEN}

En los últimos quince años, y cada vez más, la logística humanitaria ha despertado el interés de investigadores en el campo de la logística y el manejo de cadenas de abastecimiento (supply chain management, SCM en inglés). Algunos de ellos consideran que este es un campo novedoso de aplicación de los métodos y las herramientas de gestión desarrollados dentro de las empresas de negocios. La investigación sobre logística humanitaria tiende con frecuencia a favorecer un enfoque técnico, particularmente con respecto del management operacional (optimización del transporte, ubicación de los centros regionales, etcétera). Aunque este punto de vista sigue vigente en cuanto al mejoramiento del aprovechamiento de recursos logísticos escasos, tiene que estar asociado a métodos organizacionales que busquen la mejor manera de coordinar las cadenas humanitarias de abastecimiento. En este artículo se adopta este punto de vista sobre el concepto de estrategia colectiva y su aplicación al sismo de Pisco (15 de agosto de 2007). La ayuda humanitaria fue distribuida por varios comités de defensa civil pero sobre todo por el Instituto Nacional de Defensa Civil (Indeci), organismo que dirigió todo el sistema. La dirección del Indeci puso énfasis en la importancia de la acción colectiva para el mejoramiento del rendimiento humanitario, pero también en las dificultades que resultan de esta acción.

Palabras claves: acción colectiva, cadena humanitaria de abastecimiento, Indeci, logística humanitaria, sismo de Pisco.

\begin{abstract}
In the last fifteen years, and increasingly every day, humanitarian logistics has awakened the interest of researches in the logistics and supply chain management (SCM) field. Some consider this is a new area to apply methods and management tools developed in the business area. Researches in humanitarian logistics tend to favor a technical approach, particularly in operational management (transport optimization, location of regional centers, etc.). Although this view is still valid regarding improvement in using scarce logistics resources, this must be associated to organizational methods that seek the best way to coordinate within humanitarian supply chains. The present article assumes this position upon the concept of collective strategy and how it was applied to the earthquake disaster in Pisco, Peru (August 15, 2007). The humanitarian aid was distributed among several disaster aid committees, but above all to the Instituto Nacional de Defensa Civil (Indeci), the state organization that supervised the whole system. Indeci's management emphasized the importance of collective action to improve the humanitarian aid operation, but it also viewed the difficulties that resulted from this action.
\end{abstract}

Keywords: Collective actions, humanitarian supply chain, INDECI, humanitarian logistics, earthquake in Pisco.

1. Asociación Civil Cooperación Logística Solidaria, Perú. Centro de Investigación en Transporte y Logística (CRET-LOG) de la Université de la Méditerranée (Aix-Marseille 2), Francia. Doctorado en Logística y Estrategia, Université de la Méditerranée (Francia).<j.chandes@ cooperacionlogisticasolidaria.org $>$.

2. Université de la Méditerranée (Aix-Marseille 2), Francia, Centro de Investigación en Transporte y Logística (CRET-LOG) de misma universidad. Doctor (Ph. D.) en Ciencias Económicas y Profesor en Ciencias de Gestión, Université de la Méditerranée (Aix-Marseille 2), Francia. < gilles.pache@univmed.fr>. 


\section{INTRODUCCIÓN}

El 26 de diciembre de 2004, en la madrugada, un terremoto submarino de una violencia poco habitual tuvo lugar en la costa de Sumatra, con una magnitud de más de 9 grados en la escala de Richter. Este movimiento telúrico produjo un tsunami que el mundo entero todavía recuerda. Afectó a Indonesia, las costas sureñas de India y Sri Lanka, el sur de Tailandia y la isla turística de Phuket. Su costo en vidas humanas fue devastador. A la fecha, no se ha podido establecer el número exacto de víctimas, pero se calcula que las cifras ascienden a 310 mil muertos. La costa de Sumatra fue abatida por enormes olas que llegaron hasta los diez metros de altura. Del otro lado del océano Índico, Somalia y las islas Seychelles se vieron también afectadas por olas de hasta 4 metros de altura, al cabo de 8 horas de la llegada del tsunami a Sumatra. Estos hechos recuerdan que nuestro planeta sufre regularmente de trastornos violentos que no perdonan a las poblaciones civiles, las cuales apenas en unos minutos pueden llegar a perder los frutos de toda una vida de trabajo o, lo que es peor, en ocasiones son despojadas hasta de la propia vida. Ayudar a los sobrevivientes es por ello una prioridad absoluta y requiere del desarrollo de operaciones logísticas muy complejas.

Organizar de manera eficaz la logística humanitaria se ha convertido hoy en un desafío económico de gran importancia. Se trata de una situación totalmente nueva. Durante mucho tiempo la preocupación prioritaria consistía en recaudar donaciones para disponer de suficientes recursos financieros y así afrontar los efectos de una catástrofe natural (terremoto o hambruna) o una catástrofe provocada por el hombre (guerra o golpe de estado). El planteamiento de un uso eficaz de esos recursos dentro de una perspectiva logística es bastante reciente. El Fritz Institute de San Francisco fue el iniciador de reflexiones minuciosas sobre la manera de abordar de un modo más "profesional" la logística humanitaria que se define como:

El proceso de planificación, ejecución y control del eficiente y rentable flujo y almacenamiento de mercancías y materiales, así como información relacionada, desde el punto de origen hasta el punto de consumo, con el fin de aliviar el sufrimiento de las personas vulnerables. La función abarca una gama de actividades, incluyendo la preparación, planificación, adquisición, transporte, almacenamiento, seguimiento y rastreo, y el despacho de aduanas (Thomas \& Kopczak, 2005, p. 2).

En este ensayo se examina la problemática de las cadenas de abastecimiento humanitario dentro de un marco conceptual que enfoca la coordinación necesaria entre los miembros de la propia cadena de abastecimiento. Después de un largo periodo de desinterés, este tema es materia de investigación por parte de la comunidad científica. Un desastre natural, o aquel causado por la mano del hombre, implican la gestión de cadenas efímeras de abastecimiento con prontitud y de manera sincronizada con la realización de los objetivos de rendimiento.

La logística de los negocios y la logística humanitaria tienen más en común de lo que se suele imaginar. Se debe reaccionar con costos controlados (ya que los recursos financieros de los donantes no son inagotables), tanto por parte de las organizaciones que ayudan a las poblaciones afectadas, como de los fabricantes o los grandes mayoristas involucrados en una guerra competitiva, aunque las "armas" usadas sigan siendo específicas. Desde este punto de vista, nuestro propósito es doble: 1) establecer claramente las dificultades que existen en la coordinación logística por la complejidad de las operaciones humanitarias y 2) sugerir estrategias para el planeamiento de una mejora en esta coordinación.

Las acciones humanitarias en situaciones de crisis se caracterizan por un nivel necesariamente alto de reacción, fiabilidad y adaptación a las necesidades de las víctimas. Aunque las organizaciones involucradas pueden haber adquirido individualmente un know how ejemplar en numerosas experiencias anteriores, tienen todavía dificultades para trabajar rápidamente en conjunto en el campo. El número creciente de actores claves presenta un problema de coordinación, pues la existencia de numerosos actores, con frecuencia con notorias diferencias en cuanto a naturaleza, tamaño y área de especialización, también hace que haya diferencias en sus modos de operación. Sin embargo, esta coordinación es una condición directa para una ayuda exitosa. Con el fin de mejorar el manejo de la asistencia humanitaria, los actores tendrán que aprender a elaborar y manejar en conjunto las cadenas de 
abastecimiento de ayuda. En otras palabras, solo una estrategia colectiva podrá mejorar los resultados de las cadenas de abastecimiento humanitario; mientras que su ausencia tendrá consecuencias dramáticas para las poblaciones afectadas. Un caso de estudio, hecho sobre el terremoto de Pisco en el Perú, ocurrido el 15 de agosto de 2007, complementa el análisis estructural y pone de relieve estos problemas.

\section{LOGÍSTICA HUMANITARIA: UN ORIGINAL ENFOQUE DE LA GESTIÓN}

En los últimos quince años, y con una importancia cada vez mayor en tiempos recientes, la logística humanitaria ha despertado el interés de los investigadores en el manejo de las cadenas de abastecimiento, supply chain management (SCM) (Kovacs \& Spens, 2007, 2008; Goldsman, 2009; Tomasini \& Van Wassenhove, 2009a). Algunos consideran que el SCM es un campo novedoso de aplicación de métodos y herramientas de gestión desarrollado dentro de las empresas. Por supuesto, es innegable que las emergencias generadas por desastres naturales o por la mano del hombre son singulares: son vidas las que están en juego. Por tanto, no se trata simplemente de procurar brindar al consumidor un servicio logístico de calidad al costo más bajo posible. La dimensión fugaz de la asistencia humanitaria, el carácter fundamental de una preparación previa y rigurosa, y la capacidad para movilizar y coordinar considerables recursos lo más pronto posible emparentan las decisiones relativas a la logística humanitaria con los principios del lean management (Pettit \& Taylor, 2007). No sorprende por ello que la investigación académica del SCM analice ahora esta cuestión en un número creciente de documentos y ediciones especiales de revistas.

\section{De un acercamiento operacional a un acercamiento organizacional}

En los diversos trabajos dedicados a la logística humanitaria se debe subrayar el lugar que ocupan las investigaciones enfocadas en los problemas de administración óptima de los recursos (transporte, almacenamiento, etcétera) e insumos disponibles para la ayuda humanitaria. Estas investigaciones intentan optimizar el uso de recursos de transporte, refiriéndose, por ejemplo, a modelos importados del contexto militar (Pettit \& Beresford, 2005; Weeks, 2007; Goldsman, 2009); pero en todos los casos emplean complejos enfoques econométricos (Dekle, Lavieri, Martin, Emir-Farinas, \& Francis, 2005; Balcik, Beamon, \& Smilowitz, 2008; Benini, Conley, Dittemore, \& Wasman, 2009; Blecken, Rottkemper, Danne, \& Hellingrath, 2009). Estos enfoques son de un interés innegable porque identifican las múltiples variables que condicionan el proceso de obtención de una decisión óptima. El rendimiento en logística humanitaria dependerá entonces en gran medida de la naturaleza del sitio en el cual ocurra el desastre, la cantidad y la concentración de personas afectadas, la calidad de la infraestructura, la cantidad de vehículos disponibles, etcétera.

Deseando alejarse de la técnica de simple optimización, algunos investigadores han trabajado la cuestión de una gestión global mejorada de la cadena humanitaria de abastecimiento mediante una ubicación previa y eficiente de recursos logísticos que sean útiles en caso de producirse un desastre. En lugar de esperar pasivamente a que una situación de crisis se presente para lanzar operaciones humanitarias, consideran mejor estar preparados y movilizar los recursos materiales o no materiales con anticipación. Por esta razón, se debe tener en cuenta la ubicación de los lugares de almacenamiento de los recursos, pues una mala ubicación aumentaría el riesgo de alargar los plazos de reacción y tendría un impacto negativo en la ejecución de la ayuda humanitaria (Hale \& Moberg, 2005; Kapucu, Lawther, \& Pattison, 2007). Mientras más rigurosa sea la preparación, más fácil será movilizar los distintos eslabones de la cadena humanitaria de abastecimiento de una manera ágil y será mejor también, y más dinámica, la organización de sus recursos y competencias. No sorprende por eso que Kovacs y Tatham (2009), en una publicación reciente acerca de los métodos para mejorar los tiempos de respuesta de las cadenas humanitarias de abastecimiento, reafirmen la importancia del almacenamiento anticipado de los bienes en centros regionales y la capacidad requerida de los diferentes actores humanitarios para poder operar en conjunto.

Sería tentador, pero a la vez arriesgado e ineficiente, pensar que cada desastre es un acontecimiento único y por tanto requiere de una respuesta hecha a la medida; 
resultaría imposible extraer una lección general para aplicarla en eventos futuros. Sin duda, el tsunami que diezmó el Sureste de Asia en diciembre de 2004 nada tuvo en común (en cuanto a características y efectos) con el terremoto en Cachemira en octubre de 2005, y menos todavía con la tragedia humanitaria de Darfur, cuya respuesta ha sido la indiferencia por parte de la opinión pública occidental. Más allá de las diferencias objetivas entre estas situaciones de crisis, lo esencial es determinar las similitudes existentes con el fin de poder acelerar la movilización de recursos y actores. La identificación de similitudes, por ejemplo en términos de recursos de transporte o sitios de almacenamiento, debe dar lugar a posteriores acciones que sigan procedimientos estandarizados. Esta es la única manera de mejorar la resiliencia de las cadenas humanitarias de abastecimiento. En un mundo inseguro, en el cual la reacción más rápida posible puede salvar miles de víctimas, este no es un tema únicamente de dinero sino que es, sobre todo, una cuestión humana.

Existe un alto riesgo en el hecho de querer elaborar esquemas de acción universales frente a los desastres, pues vuelve muy rígidas las cadenas humanitarias de abastecimiento, al reproducir de forma automática e inapropiada los mismos patrones logísticos en todas las crisis que se presenten. Un experto en logística de Médecins Sans Frontières pone énfasis en la falta de una contextualización al criticar la estandarización excesiva de los procedimientos que emplean las cadenas de abastecimiento (Maïola, 2007). Evocando la experiencia del terremoto de Cachemira en octubre de 2005, señala los errores de Médecins Sans Frontières Bélgica, por causa del desconocimiento de las zonas de clima frío. Únicamente estaba disponible un equipo estándar, sobre todo en cuanto a la ropa, pero era totalmente inapropiado para la situación. Los pedidos debieron ser programados con urgencia y la falta de bienes demoró su entrega con consecuencias dramáticas para la población afectada. Este autor concluye que el ambiente operacional de la asistencia humanitaria es un ambiente en constante cambio y, por lo tanto, es imposible llegar a un estandarización completa de los procedimientos logísticos.

\section{¿Cómo adaptar los conceptos de gestión a la logística humanitaria?}

Lamentablemente, muchos técnicos de organizaciones humanitarias están convencidos de que cada intervención es única y no puede compararse con las demás. Un ejemplo aleccionador es el testimonio de un director de logística que fue hombre de acción por muchos años antes de cumplir funciones administrativas de primer nivel. Para entender con claridad en qué consiste la logística humanitaria, a partir de su propia experiencia, escribió:

\footnotetext{
Existen, por supuesto, reglas y principios generales, un acuerdo, políticas y competencias que nos guían en nuestro trabajo. No lo realizamos simplemente impulsados por nuestra afición a la improvisación o por nuestra capacidad para adaptarnos al medio ambiente. Cada intervención es específica y no se debe actuar nunca como si se tratase de una costumbre. Toda misión debe ser considerada como una primera misión (Diaz, 2006, p. 46).
}

Sin embargo, esta posición extrema, compartida por muchos, subestima que la técnica más apropiada es saber estandarizar aquellos procedimientos cuyas características comunes han sido identificadas en cada desastre; mientras, por otro lado, se mantiene la libertad completa de adaptar algunos elementos de la cadena humanitaria de abastecimiento en función del contexto geográfico, la calidad de la infraestructura, la situación geopolítica, el grado de daño sufrido por la población, etcétera. En otras palabras, se debe establecer el punto ideal de desvinculación entre la estandarización (invariables universales) y la adaptación (realidades locales) para poder movilizar los recursos del modo más eficaz y considerar las características específicas de la asistencia humanitaria que deberá enviarse en un momento dado en un terreno determinado. Como ya se ha señalado, esta técnica de logística de empresa capta particularmente la atención de numerosos investigadores, quienes la consideran esencial para lograr la creación de una cadena humanitaria de abastecimiento ágil (Oloruntoba \& Gray, 2006; McLachlin \& Larson, 2009).

En resumen, toda cadena humanitaria de abastecimiento tiene que ser capaz de reaccionar rápidamente ante los cambios imprevisibles del medio ambiente cuando la incertidumbre de la demanda es elevada, 
mucho más elevada que la relativa a los productos de consumo destinados a los consumidores. El Cuadro 1, inspirado en la contribución de Charles (2008), retoma los cinco componentes tradicionales del servicio ágil aplicándolos al contexto humanitario. El interés de este cuadro radica en identificar los aspectos sobre los cuales es necesario insistir y las soluciones que se han encontrado a partir de estos. Por ejemplo, el mejor grado de reacción de la cadena humanitaria se basa hoy en la creación de equipos de supervivencia modular: "kits de supervivencia" que integran elementos universales a los cuales se han añadido elementos específicos para cada catástrofe en función del clima, las creencias religiosas, los tabúes sexuales, etcétera. Desde este punto de vista, conocer tanto el abastecimiento humanitario como el abastecimiento empresarial permite encontrar soluciones de alto rendimiento.

A partir de esta reciente evolución, se podría esperar que el abastecimiento humanitario conozca hoy una relativa madurez que permita contemplar su análisis dentro de una perspectiva ya no solo operativa sino también estratégica. Así, la revista académica dirigida por Kovacs y Spens (2008) muestra que la mayoría de las investigaciones se interesan en la etapa de respuesta inmediata a la catástrofe dentro de una perspectiva operativa. Efectivamente, una nueva corriente, aún marginal, trata de estudiar los procesos de planificación con el objetivo de colocar con antelación los recursos de ayuda y reaccionar con mayor rapidez en situaciones de emergencia humanitaria, pero las reflexiones no integran una dimensión interorganizacional que es, sin embargo, esencial. En otras palabras, los investigadores no se preocupan por los problemas de organización entre los diferentes actores, mientras que para Kovacs y Spens (2008) estos condicionan directamente el rendimiento eficaz de la acción.

La fragmentación extrema de las intervenciones de las organizaciones humanitarias durante y después de la catástrofe sigue siendo la realidad más común (Oloruntoba, 2005). Cada organización tiende a manejar su propio sistema de abastecimiento, el que ha construido a lo largo de los años, y la emergencia de la

Cuadro 1. Los Cinco Componentes de una Cadena de Abastecimiento Humanitario Ágil

\begin{tabular}{|c|c|c|}
\hline Componente & ¿Por qué? & ¿Cómo? \\
\hline Flexibilidad del volumen & $\begin{array}{l}\text { El volumen depende de las donaciones, general- } \\
\text { mente impredecibles. Depende también de las ne- } \\
\text { cesidades de los destinatarios, conocidas solo des- } \\
\text { pués de la catástrofe y que varían conforme pasa el } \\
\text { tiempo. }\end{array}$ & $\begin{array}{l}\text { - Envío de equipos de expertos preentrenados } \\
\text { en las primeras } 24 \text { horas para identificar las } \\
\text { necesidades. } \\
\text { - Estandarización de todos los productos de } \\
\text { urgencia que pueden serlo. }\end{array}$ \\
\hline Flexibilidad de la entrega & $\begin{array}{l}\text { Poca o ninguna visibilidad sobre los programas de } \\
\text { entrega, que son modificados según la llegada de } \\
\text { promesas de donaciones. }\end{array}$ & $\begin{array}{l}\text { Desarrollo de sistemas y procedimientos para } \\
\text { administrar las donaciones, fichas con el detalle } \\
\text { de los puestos para el personal que gestiona los } \\
\text { contactos con los donantes y el terreno. }\end{array}$ \\
\hline $\begin{array}{l}\text { Flexibilidad del sistema } \\
\text { de oferta }\end{array}$ & $\begin{array}{l}\text { Gran diversidad de productos por administrar, se- } \\
\text { gún el tipo, el lugar y el momento de la crisis huma- } \\
\text { nitaria. }\end{array}$ & $\begin{array}{l}\text { Creación de sistemas informáticos hechos a } \\
\text { la medida que permitan editar los cuadros de } \\
\text { movilización para cada crisis. }\end{array}$ \\
\hline $\begin{array}{l}\text { Reacción de la cadena } \\
\text { logística }\end{array}$ & $\begin{array}{l}\text { Necesidad de poner rápidamente en marcha la ca- } \\
\text { dena logística y hacerla evolucionar según las ne- } \\
\text { cesidades mediante el desarrollo de herramientas y } \\
\text { métodos adaptados. }\end{array}$ & $\begin{array}{l}\text { - Almacenamiento previo de stocks para } \\
\text { las necesidades vitales básicas ("kits de } \\
\text { supervivencia", carpas, etcétera). } \\
\text { - Firma de acuerdos anticipados con los } \\
\text { proveedores. }\end{array}$ \\
\hline $\begin{array}{l}\text { Flexibilidad del portafolio } \\
\text { de productos }\end{array}$ & $\begin{array}{l}\text { La evaluación de las necesidades se realiza paralela- } \\
\text { mente a la puesta en marcha de la cadena logística, } \\
\text { lo que hace variar la naturaleza de los productos en } \\
\text { el tiempo. }\end{array}$ & $\begin{array}{l}\text { Establecimiento de un catálogo completo de } \\
\text { los diversos productos disponibles. }\end{array}$ \\
\hline
\end{tabular}

Fuente: Charles, 2008.

Elaboración propia. 
intervención hace que sea difícil coordinar los recursos materiales y humanos con las otras organizaciones. Sin embargo, no se puede mejorar la acción humanitaria con el objetivo de salvar vidas sin hacer un esfuerzo de integración logística, es decir, comprender previamente cada sistema logístico y sus interacciones en busca de una gestión más eficaz de las interconexiones. Por ejemplo, sin integración logística, cada organización humanitaria corre el riesgo de entrar en competencia con las otras organizaciones para adquirir medios de transporte; con esto se puede aumentar enormemente el precio de este lo que, al final, producirá despilfarro de los recursos financieros (Lu, Pettit, \& Beresford, 2006). Para mejorar la acción es indispensable tener una visión colectiva.

\section{Aportes de un análisis en términos de estrategia colectiva}

Skoglund (2009) sugiere analizar la acción humanitaria en tres niveles diferentes. El nivel estratégico debe conducir a indicar claramente qué organización está implicada en qué actividad para evitar cualquier redundancia costosa: buscar sobrevivientes en la zona de un terremoto, traer ayuda material a las víctimas, asegurar la llegada de víveres y medicinas, etcétera. El nivel táctico consiste en organizar en el terreno cada una de las actividades de manera eficaz por medio de los métodos adecuados; por ejemplo, la técnica mejor adaptada a un determinado terreno para encontrar sobrevivientes bajo los escombros. Finalmente, el nivel logístico corresponde a las actividades de apoyo puestas inmediatamente al servicio tanto de los rescatistas como de los sobrevivientes, con el fin de optimizar la acción humanitaria: recursos materiales adecuados para el terreno, medios de comunicación entre los equipos, etcétera. Estos tres niveles son complementarios pero no corresponden a los mismos horizontes temporales e iguales objetivos: una planificación de largo plazo para la estrategia, una conducción de mediano plazo para la táctica y una reacción de corto plazo para la logística.

Queda claro que los niveles estratégico y táctico se refieren a los modos de ajuste entre los diferentes actores de la acción humanitaria que deben ponerse en marcha para aumentar la eficacia del nivel logísti- co. Mientras mayor sea el número de actores, más susceptible de aumentar será, a su vez, la complejidad de los ajustes entre ellos, como consecuencia de la multiplicación de las conexiones operacionales. De esto se deduce un punto fundamental: la acción en el terreno es primero de naturaleza colectiva y debe responder al desafío de la coordinación interorganizacional (Rey, 1999; Majchrzak, Jarvenpaa, \& Hollingshead, 2007). Esta será aún más eficaz si la calidad de los ajustes mutuos entre los actores alcanza un alto nivel; de no ser así, las disfunciones con respecto de la disponibilidad de los recursos corren el riesgo de tener un impacto dramático sobre las poblaciones siniestradas. Esto es tan válido en un contexto de catástrofe humanitaria como en todo ecosistema: cada organización está obligada a adaptarse rápidamente a un entorno más o menos inestable, en función de sus recursos y capacidades.

En opinión de Astley y Fombrun (1983), la escasez de capacidades organizacionales y la falta de recursos para enfrentar las dificultades detectadas por las organizaciones constituyen el germen de la formulación de estrategias colectivas; por ejemplo, estabilizar el proceso de suministro, reducir la competencia y enfrentar una oportunidad de negocio. Estos autores consideran que la turbulencia y la complejidad del ambiente refuerzan este requerimiento absoluto de acción colectiva y encuentran eco en el contexto humanitario. Por lo general, el ambiente de las organizaciones es aún más complejo debido a que sus elementos están fuertemente interconectados y son interdependientes, pero actúan de manera individual y a veces contradictoria. Para protegerse de amenazas externas y perpetuar su existencia a través de un alto rendimiento, las organizaciones tienen que desarrollar una construcción colectiva de su ambiente, una especie de ambiente negociado (Cyert \& March, 1963). En él, las estrategias colectivas parecen ser un medio eficiente para manejar las interdependencias necesarias entre las organizaciones; pues tienen el poder de limitar la turbulencia del ambiente al dar coherencia a las acciones de los miembros de la comunidad (Bresser \& Harl, 1986).

Astley y Fombrun (1983) presentan las estrategias colectivas como estrategias voluntaristas, ya que tienden a comprobar que las organizaciones más dinámicas pueden controlar su destino de manera colectiva. 
Las estrategias colectivas, basadas en compartir recursos, también tienen un propósito económico puesto que ayudan a limitar el impacto de las turbulencias generadas por las muchas, y a veces contradictorias, acciones independientes de cada organización. Este es un asunto crucial en la acción humanitaria, en la cual, como ya se ha indicado, la falta de coordinación entre distintas ONG, o entre las ONG y el sector privado, puede paralizar el funcionamiento de las cadenas de abastecimiento. Estos autores identifican cuatro estrategias colectivas y distinguen dos elementos en función de los posibles tipos de asociación. Por un lado: 1) asociaciones comensales (entre organizaciones competitivas dentro de una relación horizontal) y 2) asociaciones simbióticas (entre organizaciones complementarias dentro de una relación vertical). Y por el otro: 3) asociaciones directas que realizan el enlace entre las organizaciones a través de acuerdos que definen los beneficios para cada socio y 4) asociaciones indirectas, formales o informales, que no especifican los beneficios económicos para los socios (Cuadro 2).

Cuadro 2. Las Cuatro Estrategias Colectivas Posibles

\begin{tabular}{ccc}
\hline TIPOS DE ASOCIACIÓN & Comensal & Simbiótica \\
\hline Directa & Confederada & Conjugada \\
Indirecta & Aglomerada & Orgánica \\
\hline
\end{tabular}

Fuente: Astley y Fombrun, 1983.

Elaboración propia.

La aparición de un centro estratégico (o hub en inglés) es un requisito para el desarrollo de una visión estratégica colectiva que sea compartida por toda la comunidad, sean empresas privadas $u$ organizaciones humanitarias las involucradas en el manejo de una cadena humanitaria de abastecimiento; sin embargo, deben conservar la autonomía necesaria para el desarrollo de cada organización en cuanto a la toma de decisiones. El centro estratégico desempeña una valiosa labor en la difusión de la visión estratégica cuya meta es proporcionar las instrucciones para el establecimiento de los planes de operación. Según Livet y Thévenot: “... la acción colectiva aparece solo cuando es posible constatar un cierto orden [...] en las acciones de varias personas..." (1994, p. 139). La coordinación de acciones colectivas se refiere a la coherencia, en realidad a la cohesión, que es un factor indispensable para evitar que se propaguen los efectos entrópicos. Una de las funciones de un centro estratégico es asegurar esta cohesión, es allí donde el centro deberá tener una vasta labor de coordinación aceptada por todos.

La legitimidad de un centro estratégico puede basarse en su propio rendimiento, sus conocimientos especializados sobre un campo determinado y su capacidad para difundir una visión colectiva y promover su aplicación entre los demás socios a través de un lenguaje claro que responda a los intereses de cada parte involucrada. Esta acción es de naturaleza temporal. Los mecanismos de coordinación entre los actores claves tienen que ser, en primer lugar, desarrollados $y$, en segundo lugar, probados. Cada actor aprende a gestionar la cooperación, las relaciones entre los socios y su evolución dinámica (Ingham, 1994). Por lo tanto, cuando la relación alcanza cierto nivel de madurez, el liderazgo del centro estratégico tiende a debilitarse. Este razonamiento se puede aplicar a la logística humanitaria en cualquier tipo de crisis. La urgencia de la intervención para salvar vidas implica, mecánicamente, que los actores claves sigan una estrategia colectiva para mejorar su reacción frente a las emergencias, condición insoslayable para alcanzar un uso eficiente de recursos escasos. Pero, ¿cuáles son las prácticas actuales en las estrategias colectivas?, ¿no existen, acaso, verdaderas dificultades para su implementación? Un estudio de caso presenta una experiencia original para explorar los elementos principales de la problemática y proporciona oportunidades interesantes para su mejor comprensión.

\section{ESTUDIO DE CASO: APLICACIÓN AL TERREMOTO DE PISCO, PERÚ}

Para este estudio de caso se emplea el método de observación participante y se apela a la perspectiva adoptada por Bogdan y Taylor (1975): la investigación se caracteriza por un extenso periodo de interacciones sociales entre el investigador y las personas investigadas en su ambiente. En efecto, dado que la gestión es materia de la ciencia de la acción, la investigación ha sido deliberadamente inscrita en una corriente epistemológica moderna nacida del pensamiento sistémico: el constructivismo. La investigación-acción encuentra sus raíces en 
la resolución de problemas concretos en un determinado contexto y la transformación de una situación vivida por los actores como problemática en una solución con mayores ventajas (Greenwood \& Levin, 2007). Partiendo del principio de que la realidad social es una construcción que se elabora en las interacciones que los individuos efectúan entre ellos y con el mundo que les rodea, la resolución de problemas se presenta como resultado de un proceso de construcción con los actores de la organización estudiada. En esta perspectiva, participantes e investigadores son todos "co-investigadores" y "co-actores" $y$ deben ponerse de acuerdo sobre el diagnóstico y las acciones a emprender. La construcción del problema procede de un aprendizaje mutuo que se desarrolla a lo largo del proceso de investigación.

Uno de los autores de este artículo es miembro de una agencia gubernamental en el Perú desde mayo de 2007, y esta agencia ha estado involucrada en el manejo de operaciones de logística humanitaria. Aprovechando su estatus de observador participante interno, este investigador pudo formar parte del quehacer diario de las operaciones de la agencia gubernamental y también pudo participar en el proceso de toma de decisiones. Esto le permitió recoger una gran cantidad de información en bruto y plasmarla en un portafolio de datos. Este papel participativo ayudó a obtener una posición privilegiada de análisis, posibilitada por una permanente dialéctica entre teoría y práctica en el terreno, durante el terremoto ocurrido en Pisco, Perú, el 15 de agosto de 2007 , con el objetivo de obtener un registro directo de algunos errores logísticos $y$, después, emitir las recomendaciones pertinentes para evitar su repetición. Optar por una investigación de ingeniería permite así, a partir de la experiencia y la realidad interpretada por los actores y los investigadores, una "co-construcción" de un modelo de gestión adaptado a la situación que pueda ser asimilado por los actores.

\section{Acerca de la metodología}

Para cumplir los objetivos de la investigación, el proceso de investigación-acción se divide en ciclos. Cada fase se caracteriza por diferentes métodos de recopilación y manejo de información y por el papel que desempeña el investigador. Así, el investigador alterna las fases de inmersión operacional y reflexión y construcción científica, lo que le permite evaluar el proceso de investigación-acción, actualizar sus valores y también hacer evolucionar el proyecto de investigación en función de la nueva información recogida y los fenómenos observados. Con el propósito de evaluar la pertinencia de las propuestas y enriquecer la reflexión mediante intercambios de ideas para perfeccionar la metodología o el contenido de la investigación, a cada fase de construcción teórica le sigue la reunión de un comité compuesto por miembros de la Dirección Nacional de Logística, la Dirección Nacional de Operaciones y la Dirección Nacional de Planificación, y el servicio informático.

Desde los primeros meses (entre abril y agosto de 2007), el investigador inició una fase denominada "exploratoria" que apuntaba a identificar a los principales protagonistas, asimilar el entorno, realizar el recuento de información y elaborar un cuaderno de ocupaciones mediante entrevistas libres. La fase de "diagnóstico" (de agosto a diciembre de 2007) debutó con el sismo de Pisco y permitió realizar un diagnóstico organizacional y logístico de la coordinación humanitaria. Este difícil periodo, dado el contexto, fue propicio para el convencimiento de la necesidad de aplicar cambios organizacionales y efectuar propuestas de soluciones (confrontación entre decisiones teóricas y fenómenos observados). La fase de "laboratorio" (de enero a agosto de 2008) fue un periodo transitorio en el cual el investigador, siempre en contacto con los actores, permaneció como observador de la evolución de un territorio inestable y fragilizado por las consecuencias políticas de la caótica gestión del acontecimiento de Pisco. Finalmente, la fase "participativa" (de setiembre a diciembre de 2008) fue la última etapa orientada a la comprobación de la pertinencia del modelo teórico elaborado en la fase de "diagnóstico". Esta etapa, alternativa a la anterior, se concretó por la Asociación Civil Cooperación Logística Solidaria, la cual tiene por misión, en un primer momento, compartir los "conocimientos accionables" con los actores que constituyen la red humanitaria y, en una segunda etapa, la puesta en marcha de la cadena logística humanitaria.

\section{La importancia de la unidad central de dirección}

El 15 de agosto de 2007, el terremoto de Pisco, en la costa peruana 200 kilómetros al sur de Lima, de una 
magnitud de 7.9 grados en la escala Richter, acabó con la vida de más de 500 personas y tuvo un impacto directo sobre la vida de otras 655 mil más, cuyas necesidades básicas de agua, alimento, abrigo y vestido, entre otras, tuvieron que satisfacerse de manera inmediata (Figura 1). En palabras de D'Ercole, Chandes, Perfettini y Audin:

El número relativamente bajo de víctimas (ampliamente inferior a las 67.000 víctimas del 15 de mayo de 1970 que afectó el norte del país) se explica por la concurrencia de diversos factores: la hora, el sismo se produjo a las 18h40, cuando la mayoría de las personas afectadas ya no se encontraba en sus centros laborales (particularmente oficinas), los colegios estaban vacíos y la población no se vio sorprendida en plena noche mientras dormía; además, el segundo sismo se produjo 60 segundos después, lo que permitió que la población evacuara sus viviendas (en su mayoría casas de uno y dos pisos) antes que éstas se derrumbaran; la ausencia de réplicas fuertes; la distancia relativamente corta (menos de $200 \mathrm{~km}$ ) que separa las zonas más afectadas de la capital del Perú: a pesar del bloqueo de las carreteras y de las dificultades iniciales para afrontar la crisis, la ayuda llegó relativamente rápido (2007, p. 4).

Debido a su cercanía al epicentro, la mayoría de las víctimas y los daños se ubicaron cerca de la costa en las provincias de Chincha, Pisco e Ica. El sismo se sintió también en el interior del país, en los Andes e inclusive en el Altiplano, donde la proporción entre damnificados y población total de los distritos suele ser muy elevada.

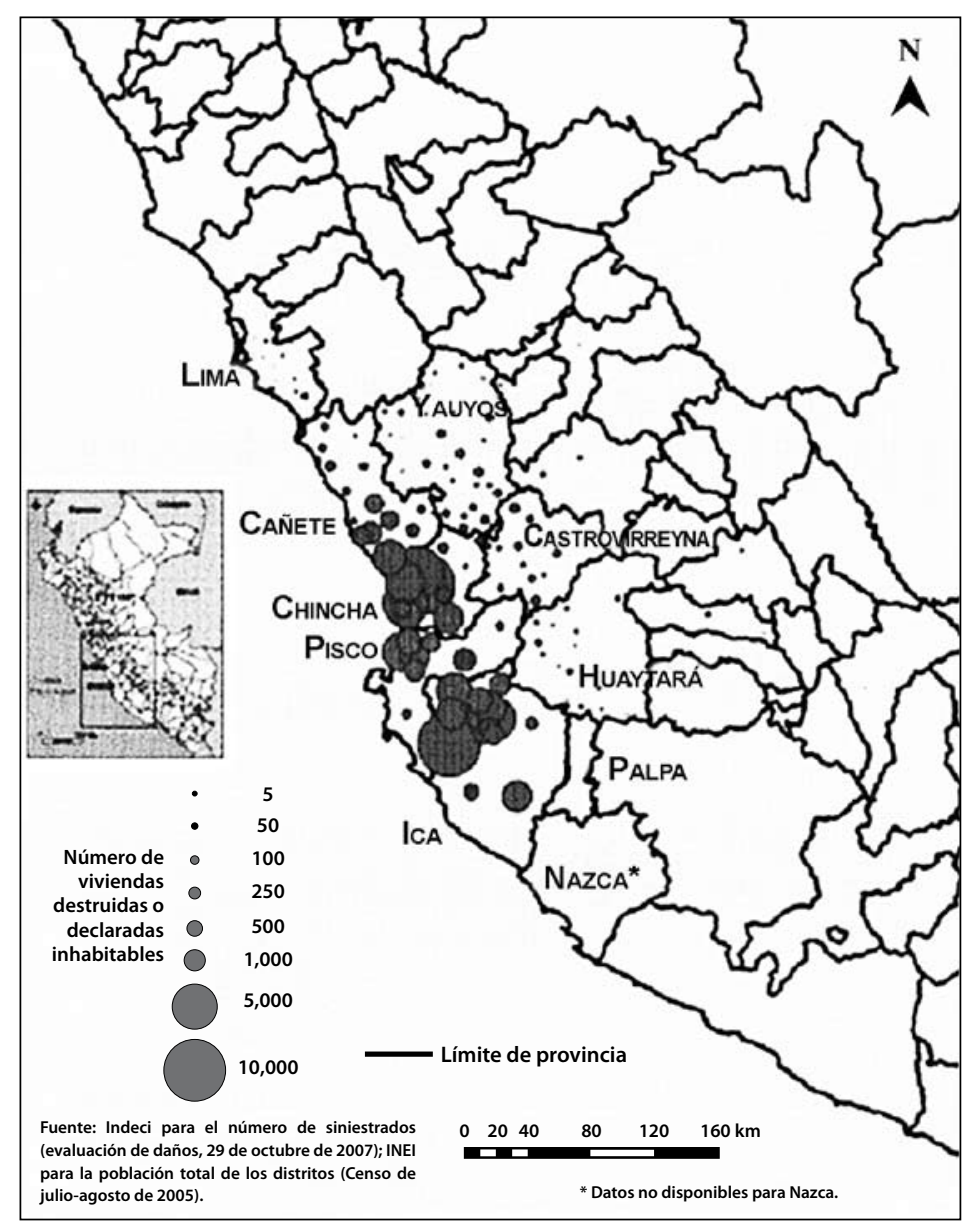

Figura 1. Los daños ligados al sismo de Pisco

Fuente: Indeci, 2007. 
La asistencia humanitaria para las víctimas fue distribuida a través de los múltiples comités de defensa civil articulados en instancias regionales, provinciales y municipales pero, sobre todo, en vista de la amplitud del desastre, por el Instituto Nacional de Defensa Civil (Indeci). Esta institución tuvo el papel de coordinadora, o central estratégica, para todo el Sistema Nacional de Defensa Civil. El Indeci reaccionó bastante rápido al enviar al Callao (zona portuaria del conglomerado limeño) equipos para evacuar a las familias que se encontraban expuestas a un posible tsunami. El 16 de agosto y los días que siguieron, otros equipos estuvieron presentes en la zona de Pisco para tratar de evaluar los daños y analizar las necesidades de la población. El 17 de agosto, Indeci organizó, junto con la Agencia Peruana de Cooperación Internacional (APCI), una mesa redonda que reunió a los representantes de las embajadas extranjeras y las organizaciones internacionales de solidaridad con el objetivo de coordinar la ayuda internacional. Asimismo, estableció, con el apoyo de la Fuerza Aérea, un puente aéreo entre el aeropuerto militar de Lima y el de Pisco el cual permitió transportar personal (médicos, rescatistas y militares, entre otros) y donaciones, es decir productos de primera necesidad como agua, víveres y frazadas. Este puente funcionó hasta el 20 de agosto, cuando se logró rehabilitar la carretera entre Lima y la zona siniestrada.

Frente a la crisis de Pisco, la defensa civil no pudo afrontar lo ocurrido y, específicamente, no fue capaz de satisfacer la necesidad de asistencia en las tres regiones impactadas. A pesar de la cantidad relativamente pequeña de muertos, en comparación con otros desastres ocurridos en el mundo, la capacidad de respuesta nacional se mostró manifiestamente inadecuada. De hecho, la cooperación internacional fue necesaria para que la ayuda llegase a la población afectada. Aunque la movilización de la comunidad en las horas y los días que siguieron al terremoto, tanto nacional como internacional, fue notable, se debe admitir que en el campo de operaciones el manejo de la logística fue más que caótico:

1) En primer lugar, la recepción y el transporte de donaciones humanitarias fueron totalmente improvisados: repetidos cuellos de botella en las bases de la Fuerza Aérea y los puertos del Callao y Pisco dieron lugar a una larga exposición a la humedad del clima invernal, un deterioro de las donaciones e, inclusive, la pérdida de algunas de ellas debido a un difícil rastreo entre el punto de llegada y el de distribución.

2) En segundo lugar, la asistencia humanitaria fue posteriormente distribuida de manera aleatoria. El resultado fue una duplicación costosa en algunas áreas, mientras que otras, más aisladas, recibieron solo un apoyo parcial. Peor aún, en la confusión que siguió al terremoto, se pudo constatar una distribución de bienes poco apropiados para las necesidades de la población o no aptos para el consumo humano (alimentos en mal estado, medicinas vencidas, etcétera).

Para entender las principales razones de estas deficiencias operacionales y logísticas asociadas con el terremoto de Pisco se deben tener en cuenta varios factores objetivos. Por un lado, como en todo desastre, las características particulares del terremoto: la naturaleza inesperada y la magnitud del evento; la diversidad y la cantidad de los bienes requeridos por las víctimas demandaban la movilización instantánea y la acción de numerosos actores. Por otro lado, era evidente que las agencias de defensa civil estaban poco preparadas: cantidades inadecuadas de productos en los almacenes; falta de recursos y material de apoyo logístico como equipos de descarga en los puertos y los aeropuertos, poca disponibilidad de medios de transporte, etcétera; debilidad de los recursos humanos para evaluar, recibir, controlar, almacenar y distribuir la asistencia humanitaria; inexistencia de un sistema informático para hacer el monitoreo sistemático y colocar los productos (tracking y tracing) a lo largo de la cadena humanitaria de abastecimiento.

Finalmente, el insatisfactorio funcionamiento del Sistema Nacional de Defensa Civil dificultó el trabajo del Indeci en los aspectos de movilización y coordinación con las diferentes organizaciones y sus recursos materiales y humanos. El resultado fue una estructuración espontánea y autónoma de la cadena humanitaria de abastecimiento, finalmente poco eficiente, y la multiplicación de acciones individuales y anárquicas de los protagonistas en el campo. 
En vista de los errores y las disfuncionalidades asociados con el manejo de la logística humanitaria después del terremoto de Pisco ¿qué desafíos enfrentan ahora el Indeci y las oficinas de defensa civil regionales? Como demostró la investigación en la primera parte de este análisis, el desafío es: 1) poder expandir de manera temporal las capacidades operacionales y logísticas y 2) garantizar la obtención y la distribución (hasta el lugar más apartado) de asistencia humanitaria apropiada para las necesidades de la población. Estos problemas tienen como común denominador el que únicamente se pueden solucionar aplicando una estrategia colectiva para evitar el riesgo de multiplicar los muertos. Los esfuerzos tienen que estar dirigidos a favorecer acuerdos de cooperación que aseguren, dentro de una organización efímera, la coordinación de una ayuda dinámica, ágil, temporal, complementaria y diversificada. Por este motivo fue creada la Asociación Civil Cooperación Logística Solidaria cuya meta es entregar una respuesta sistémica a un grupo de actores que deben aprender a trabajar juntos frente a la urgencia generada por un desastre.

Se confirmó así que para realizar una acción humanitaria es necesario basarse en equipos de almacenamiento logístico cuya misión consiste en formalizar previamente la preparación de los operativos para el caso de una intervención y gestionar después el auxilio en el momento de la catástrofe (Jahre \& Jensen, 2009). En otras palabras, el equipo de almacenamiento logístico está al servicio de una línea de acción que coordina de manera transversal a todas las organizaciones humanitarias implicadas cuyas responsabilidades se clasifican en tres niveles: la recopilación y la difusión de la información, el uso coordinado de los recursos logísticos y la formación del personal a cargo de la logística. El equipo de almacenamiento logístico debe, por lo tanto, constituir la autoridad estratégica de referencia (el centro estratégico o hub que se mencionó anteriormente) que todos los actores deberán consultar para definir su campo de intervención y evitar así las zonas de tensión. Es necesario que, desde el inicio, el equipo de almacenamiento de datos posea una legitimidad incuestionable, basada sobre todo en su capacidad de coordinar las intervenciones de los actores, las actividades y los recursos. Como resulta evidente, el principal problema radica en la posible burocratización ligada a la separación entre el estrato central (el equipo de almacenamiento de datos) y los estratos locales (las organizaciones humanitarias, las autoridades) que se enfrentan a la realidad sobre el terreno. Al atribuirle a una autoridad estratégica la responsabilidad de planificar y coordinar los operativos se corre el riesgo de ahondar la brecha entre los que deciden y los que actúan, cuando estos últimos poseen un saber a veces no formalizado pero invalorable (MacRae, 2008). Se trata, sin duda, del principal desafío que deberá afrontar la acción humanitaria en los próximos años.

Los ejemplos de Sri Lanka y Tailandia después del tsunami del 26 de diciembre de 2004 ponen de relieve la férrea voluntad por parte de los actores claves de implementar un centro estratégico con un amplio poder de decisión. Con el tsunami, Sri Lanka vivió la peor catástrofe natural de su historia y las autoridades han aprendido la lección fruto de la total desorganización de la ayuda humanitaria. Crearon un centro nacional de operaciones con la misión específica de elaborar e implementar procedimientos estándar para responder ante una emergencia (Ratnasooriya, Samarawickrama, \& Imamura, 2007). De manera más amplia, se ha establecido un proceso de institucionalización de la logística humanitaria con la aprobación del Disaster Management Act (Ley de Gestión ante Desastres) de mayo del 2005. Esta ley precisa un conjunto de directivas que deben aplicarse cuando ocurre un desastre natural. De igual manera, Tailandia ha creado una unidad central de dirección vinculada con el jefe de gobierno cuyo objetivo es facilitar la coordinación de la logística humanitaria articulada en dos niveles: 1) encargarse de la preparación y la preubicación de recursos para enfrentar cualquier eventualidad y 2) desempeñar un papel de mando si ocurre una catástrofe (Weerawat, 2007).

\section{Sugerencias para mejorar la acción colectiva}

El objetivo prioritario de la puesta en marcha de una cadena logística humanitaria de abastecimiento es llevar alivio a la población afectada. El usuario final es el que sirve como referente para determinar si la cadena de alivio ha alcanzado un nivel deseado de eficiencia. Las organizaciones involucradas en la cadena tienen que actuar de manera óptima, en el momento adecuado y con coherencia al determinar las necesidades prioritarias y 
evitar la duplicación y el desperdicio de los recursos movilizados. Cada organización tiene un campo específico de pericia y acción que, generalmente de manera individual y compartimentada, ofrece un servicio relativamente especializado en relación con su naturaleza y vocación. Ninguna tiene la capacidad o las competencias requeridas para solucionar todos los problemas que se generan en una situación de emergencia. Una circunstancia agravante son los obstáculos que encuentra la logística humanitaria con las autoridades locales quienes, debido a un deseo de autonomía, algunas veces enfrentan con mucha dificultad el aspecto intrusivo, por así decirlo, de las acciones de las asociaciones humanitarias. Bajo estas condiciones es difícil encontrar soluciones óptimas a múltiples resistencias políticas y culturales, lo cual lleva a cuestionar la pertinencia de modelos econométricos que no tratan este importante hecho (Altay \& Green III, 2006).

Para enfrentar con éxito la necesidad de una acción colectiva basada en la movilización de potenciales complementarios se sugiere una estructura virtual de coalición en la cual todos los actores claves involucrados en la etapa humanitaria o de desarrollo se unan para alcanzar el desafío de construir una cadena humanitaria de abastecimiento temporal. Esta coalición está marcada por un horizonte de corto o mediano plazo pues, una vez que los bienes o los servicios sean entregados, la coalición queda en suspenso a la espera de una próxima operación. La construcción de una cadena humanitaria de abastecimiento temporal requiere de la implementación de una infraestructura apropiada, lo que supone dos aspectos a tomar en cuenta: 1) la distancia espaciotemporal entre los socios y 2) la fusión necesaria entre los intereses individuales y colectivos de estos. Se requeriría la supervisión de un centro estratégico para facilitar que los miembros de la cadena humanitaria de abastecimiento compartan documentos, comuniquen sus planes de acción y mejoren la coordinación mutua. Para lograrlo, la organización virtual deberá emplear un sistema de información que ofrezca la posibilidad de implementar rápidamente una plataforma capaz de unir a cada organización para llegar al intercambio certero de información. A partir de la obra de Astley y Fombrun (1983), se pueden distinguir dos tipos de asociaciones colectivas para evitar la desafortunada experiencia de Pisco:
1) Una asociación simbiótica directa dentro una relación vertical. Esto lleva a la constitución de una estructura de coalición que une a dos tipos de organizaciones para asegurar una mejor articulación entre lo que se ofrece y las necesidades reales de la población por medio de la entrega de recursos y provisiones en buenas condiciones, en las cantidades requeridas, y el lugar y el momento en los que se les demande: 1) hacia arriba, los proveedores de productos humanitarios (fabricantes, donantes, organizaciones internacionales, etcétera) y 2) hacia abajo, los actores de defensa civil y las ONG. El diseño y la gestión de una cadena de abastecimiento son temporales; no obstante, alcanzan el ideal de excelencia logística descrita por Fabbe-Costes: "Una cadena logística es un arreglo temporal, efímero, que responde a una demanda o realiza una propuesta colectiva. Una cadena aparece como una activación, una estructura por proyecto. Una vez cumplida [...] la cadena desaparece." (2005, p. 156).

2) Una asociación comensal directa dentro una relación horizontal. Esto lleva a la formación de una confederación de empresas privadas y entidades especializadas en logística que negocian y se unen temporalmente para movilizar y coordinar los recursos humanos y materiales con el objetivo de responder a las necesidades logísticas frente a un desastre de dimensiones mayores. El trabajo en conjunto de las distintas organizaciones que ofrecen la misma clase de productos y servicios deriva de un propósito compartido desde el inicio: valorizar la competencia logística para satisfacer colectivamente las necesidades de las víctimas. Un propósito compartido desde el inicio ofrece la unión rápida, flexible y transicional de competencias, recursos humanos y aptitudes de cada socio. En nuestra opinión, la Asociación Civil Cooperación Logística Solidaria, como centro estratégico, tiene que desempeñar un papel de intermediario, tal como propone el modelo de 4PL investigado por Fulconis y Paché (2005), en la definición conjunta de los procesos logísticos y la mezcla de bienes tangibles (materiales) e intangibles (el saber). 
Para incrementar la visibilidad en la cadena humanitaria de abastecimiento, la Asociación Civil Cooperación Logística Solidaria tendrá que intercambiar cada vez más información para anticipar, adaptar y evaluar (monitoreo de donaciones, niveles de recursos almacenados, estatus de capacidades y recursos movilizados, etcétera). La implementación de una herramienta informática de decisiones logísticas supondrá un reforzamiento en la intermediación de la asociación y en la coordinación coherente de la cadena de alivio; esto se vincularía a un marco de knowledge management para identificar las necesidades de información y proveer a aquellos que toman las decisiones con recomendaciones útiles, basadas en experiencias del pasado (Zhang, Zhou \& Nunamaker, 2002). Un intento de aplicación del knowledge management tiene una importancia particular para mejorar la acción humanitaria y aprovechar de manera eficaz la experiencia ganada, después de un proceso de codificación. Una investigación realizada dentro de Médecins Sans Frontières Holanda muestra cuáles son las ventajas de un sistema informático de acopio, tratamiento y almacenamiento de datos relacionados con una catástrofe (Van der Laan, De Britto, \& Vermaesen, 2007). Las cuestiones más relevantes están relacionadas con la posibilidad del libre acceso a la información a través de un portal en Internet; simultáneamente, se debe considerar la cuestión del acceso a los antecedentes históricos de las acciones humanitarias, la interacción entre los expertos en logística para compartir sus experiencias y fracasos y la disponibilidad de recursos en línea.

Se puede apreciar que el manejo de la logística humanitaria no se puede reducir a la optimización del uso de una variedad de equipos y recursos. De hecho, lo primero es reflexionar sobre cómo las acciones de cada actor deberán articularse de manera coherente con una estrategia colectiva claramente establecida. La estrategia tiene que estar formulada mucho antes de cualquier emergencia para evitar la improvisación y sus dramáticos efectos, los cuales recaen sobre las poblaciones afectadas. El asunto más controvertido sigue siendo la movilización de recursos y, por ende, la inversión en coordinación logística que podría ocasionalmente, o quizá nunca, ser usada en algún lugar del mundo. Resulta que la logística humanitaria se encontrará siempre frente a la necesidad de una mejor coordinación entre actores.
De hecho, si las empresas privadas tienen interés en coordinar para incrementar sus rentas, la noción de renta está ausente en el contexto humanitario (Tomasini \& Van Wassenhove, 2009b). Por lo tanto, otras acciones son necesarias para instar a las asociaciones a colaborar y compartir información. Se trata aquí de un desafío mayor, todavía más difícil de lograr debido a que una emergencia está caracterizada siempre por la complejidad y la incertidumbre.

\section{CONCLUSIÓN}

Se entiende, desde muchos años atrás, que la acción humanitaria tiene un significado invalorable para la conciencia de los países de Occidente. ¿De hecho, cómo es posible negar algunos millones de dólares cuando las vidas de cientos de miles de personas están en juego? Lastimosamente, en un mundo de recursos insuficientes y, a pesar de no tener precio, la asistencia humanitaria sí tiene un costo. Un mejor manejo de este costo podría influir en la posibilidad de enviar ayuda a un número variable de sitios de operación. Dentro de los ahorros que pueden hacerse, la mejor coordinación logística tiene un papel determinante, por ejemplo, para evitar el envío de material inútil o excesivo alimento a un determinado lugar, mientras que ambos recursos hacen falta en sitios aledaños. Si seguimos el razonamiento de Thomas y Kopczak (2005), la logística humanitaria se enfrenta finalmente a dificultades aún mayores a las cuales debe aportar solución. Una colaboración muy limitada entre organizaciones humanitarias constituye una de esas dificultades, pero se debe añadir a esto la falta de reconocimiento de la importancia de la logística, la carencia de profesionalismo de los equipos, la falta de empleo de tecnología (en particular en lo que a sistemas de información se refiere) o, aún más, dificultades para apropiarse de los procesos de aprendizaje organizacional.

Desgraciadamente, por causa del sistema de financiamiento de las organizaciones humanitarias que aportan recursos después del acontecimiento, estas organizaciones se encuentran en situación de competencia entre sí, y cada una de ellas estaría feliz de cooperar pero... en sus propios términos. Adoptar una visión verdaderamente estratégica de la logística humanitaria requiere de una 
firme acción colectiva, en particular de los equipos de almacenamiento logísticos que sirven de hub. Para resumir, ha llegado el momento de fijar un horizonte menos idealista de la asistencia humanitaria por intermedio de la integración de técnicas de gestión para optimizar su impacto. Tomar lo mejor de la logística empresarial ya no es un tabú sino una absoluta necesidad. Nuestro planteamiento apunta a sugerir nuevas aperturas al integrar el modelo de estrategias colectivas desarrollado por Astley y Fombrun (1983) y se ha inspirado en el manejo de operaciones después del terremoto de Pisco. Como es obvio, no es más que una reflexión exploratoria que exige mayor profundidad en el manejo del campo de la logística humanitaria y mayor rigor del SCM.

\section{REFERENCIAS}

Altay, N., \& Green III, W. (2006). OR/MS research in disaster operations management. European Journal of Operational Research, 175(1), 475-493.

Astley, W., \& Fombrun, C. (1983). Collective strategy: Social ecology of organizational environments. Academy of Management Review, 8(4), 576-587.

Balcik, B., Beamon, B., \& Smilowitz, K. (2008). Last mile distribution in humanitarian relief. Journal of Intelligent Transportation System, 12(2), 51-63.

Benini, A., Conley, C., Dittemore, B., \& Waksman, Z. (2009). Survivor needs or logistical convenience? Factors shaping decisions to deliver relief to earthquakeaffected communities, Pakistan 2005-06. Disasters, 33(1), 110-131.

Blecken, A., Rottkemper, B., Danne, C., \& Hellingrath, B. (2009). A typology of operations research methods in humanitarian logistics. II International Humanitarian Logistic Symposium. Faringdon, UK.

Bogdan, R., \& Taylor, S. (1975). Introduction to qualitative research methods: A phenomenological approach to the social sciences. Nueva York: John Wiley \& Sons.

Bresser, R., \& Harl, J. (1986). Collective strategy: vice or virtue? Academy of Management Review, 11(2), 408-427.

Charles, A. (2008). Apprentissage croisé entre secteur humanitaire et industriel pour une meilleure gestion des chấnes logistiques. IX Congrès de Ecole Doctorale Systèmes (EDSYS). Toulouse: Laboratoire d'Analyse et d'Architecture des Systémes, Centre Nationale de la Recherche Scientifique (LAAR-CNRS).

Cyert, R., \& March, J. (1963). A behavioral theory of the firm. Englewood Cliffs, NJ: Prentice-Hall.
Dekle, J., Lavieri, M., Martin, E., Emir-Farinas, H. \& Francis, R. (2005). A Florida county locates disaster recovery centers. Interfaces, 35(2), 133-139.

D'Ercole, R., Chandes, J., Perfettini, H. \& Audin, L. (2007). Le séisme de Pisco du 15 août 2007: entre urgence et reconstruction. EchoGéo [Sur le vif]. URL: <http:// echogeo.revues.org/index2109.html>. Consultado el 29 de mayo de 2009.

Diaz, F. (2006). Le logisticien humanitaire. Les Tribunes de la Santé, 10, 43-50.

Fabbe-Costes, N. (2005). La gestion dynamique des supply chains des entreprises virtuelles. Revue Française de Gestion, 156, 151-166.

Fulconis, F., \& Paché, G. (2005). Piloter des entreprises virtuel- les: quel rôle pour les prestataires de services logistiques? Revue Française de Gestion, 156, 167-186.

Goldsman, D. (2009). Humanitarian logistics and supply chains: Case studies and research issues. Boca Raton, FL: CRC Press.

Greenwood, D., \& Levin, M. (2007). Introduction to action research: social research for social change (2. a ed.). Londres: Sage.

Hale, T., \& Moberg, C. (2005). Improving supply chain disaster preparedness: A decision process for secure site location. International Journal of Physical Distribution \& Logistics Management, 35(3), 195-207.

Ingham, M. (1994). L'apprentissage organisationnel dans les coopérations. Revue Française de Gestion, 97, 105-121.

Jahre, M., \& Jensen, L.-M. (2009). Supply chain design and coordination in humanitarian logistics through clusters. 
II International Humanitarian Logistic Symposium. Faringdon, UK.

Kapucu, N., Lawther, W., \& Pattison, S. (2007). Logistics and staging areas in managing disasters and emergencies. Journal of Homeland Security and Emergency Management, 4(2), 1-17.

Kovacs, G., \& Spens, K. (2007). Humanitarian logistics in disaster relief operations. International Journal of Physical Distribution \& Logistics Management, 37(2), 99-114.

Kovacs, G., \& Spens, K. (2008). Humanitarian logistics revisited. En J. S. Arlbjørn, A. Halldórsson, M. Jahre \& K. Spens (Eds.). Northern lights in logistics and supply chain management (pp. 217-232). Copenhague: Copenhagen Business School Press.

Kovacs, G., \& Tatham, P. (2009). Responding to disruptions in the supply network - From dormant to action. Journal of Business Logistics, 30(2). En prensa.

Livet, P., \& Thévenot, L. (1994). Les catégories de l'action collective. En A. Orléan (Ed.). Analyse économique des conventions (pp. 139-167). París: Presses Universitaires de France.

Lu, D. K., Pettit, S., \& Beresford, A. (2006). Critical success factors for emergency relief logistics. Whampoa: An Interdisciplinary Journal, 51(1), 177-184.

MacRae, G. (2008). Could the system work better? Scale and local knowledge in humanitarian relief. Development in Practice, 18(2), 190-200.

Maïola, A. L. (2007). Médecins Sans Frontières et la gestion des catastrophes naturelles: standardisation et limites de la logistique en situation d'urgence. Mondes en Développement, 137, 81-88.

Majchrzak, A., Jarvenpaa, S., \& Hollingshead, A. (2007). Coordinating expertise among emergent groups responding to disasters. Organization Science, 18(1), 147-161.

McLachlin, R., \& Larson, P. (2009). Pre-positioning in humanitarian logistics: A postponement strategy? II International Humanitarian Logistic Symposium. Faringdon, UK.

Oluruntoba, R. (2005). A wave of destruction and the waves of relief: Issues, challenges and strategies. Disaster Prevention and Management, 14(4), 506-521.

Oloruntoba, R., \& Gray, R. (2006). Humanitarian aid: An agile supply chain? Supply Chain Management: An International Journal, 11(2), 115-120.
Pettit, S., \& Beresford, A. (2005). Emergency relief logistics: An evaluation of military, non-military and composite response models. International Journal of Logistics: Research \& Applications, 8(4), 313-331.

Pettit, S., \& Taylor, D. (2007). Humanitarian aid supply chain assessment: A preliminary consideration of the relevance of lean supply chain concepts to humanitarian aid supply chains. XIX Nordic Logistics Research Network (NOFOMA) Conference. Reykjavik, Islandia.

Ratnasooriya, H., Samarawickrama, S. \& Imamura, F. (2007). Post tsunami recovery process in Sri Lanka. Journal of Natural Disaster Science, 29(1), 21-28.

Rey, F. (1999). The complex nature of actors in humanitarian action and the challenge of coordination in reflections on humanitarian action. Londres: Pluto.

Skoglund, P. (2009). What is humanitarian logistics? A comparison between humanitarian, business and military logistics. Il International Humanitarian Logistic Symposium. Faringdon, UK.

Thomas, A., \& Kopczak, L. (2005). From logistics to supply chain management: The path forward in the humanitarian sector. San Francisco, CA: Fritz Institute.

Tomasini, R., \& Van Wassenhove, L. (2009a). Humanitarian logistics. Nueva York: Palgrave Macmillan.

Tomasini, R., \& Van Wassenhove, L. (2009b). From preparedness to partnerships: Case study research on humanitarian logistics. International Transactions in Operational Research, 16(5), 549-559.

Van der Laan, E., De Britto, M., \& Vermaesen, S. (2007). Logistics information knowledge management issues in humanitarian aid organizations. Erasmus Research Institute of Management, ERIM Report Series ERS-2007-026-LIS. Rotterdam, Holanda: Erasmus University.

Weeks, M. (2007). Organizing for disaster: Lessons from the military. Business Horizons, 50(6), 479-489.

Weerawat, W. (2007). Efficacy of international standards on logistics in disaster management. Case study: National Crisis Management Center, Thailand. XVIII Production and Operations Management Society (POMS) Conference. Dallas, Texas.

Zhang, D., Zhou, L., \& Nunamaker Jr., J. (2002). A knowledge management framework for the support of decision making in humanitarian assistance/disaster relief. Knowledge and Information Systems, 4(3), 370-385. 
